\title{
PRIORIDADES DE INVESTIGAÇÃO EM SAÚDE MENTAL E A TRANSFORMAÇÃO DO MODELO ASSISTENCIAL
}

\author{
| Claudia de Melo Tavares ${ }^{1}$; Marcela Muniz ${ }^{2}$; Thiago Silva ${ }^{3}$ |
}

\section{RESUMO}

CONTEXTO: Nas duas últimas décadas o Brasil passou por um processo de transformação do seu modelo de atenção em saúde mental, caracterizado pelo redirecionamento de um cuidado antes centrado na internação hospitalar para uma lógica de atenção focada em serviços de base comunitária.

OBJETIVO(S): Este estudo teve como objetivo analisar a produção de conhecimento em Saúde Mental por parte dos enfermeiros do Brasil no que se refere às temáticas prioritárias da Agenda de Saúde Mental.

METODOLOGIA: Buscou-se informações contidas em artigos, em língua portuguesa indexados nas bases de dados científicos. Para contextualizar o tema, foi feito um levantamento também a respeito da distribuição dos serviços de saúde mental no Brasil.

RESULTADOS: A partir de uma busca na base de dados, foram encontrados 54 artigos. Dentre eles, pôde-se notar que há no Brasil uma produção desigual no que diz respeito ao volume de pesquisas em enfermagem de Saúde Mental para cada região do país. Propõe-se que os enfermeiros busquem sintonizar suas pesquisas às temáticas priorizadas na Agenda de Saúde Mental. A articulação em torno da Agenda é a ação mais importante na legitimação deste instrumento na Política Nacional de Ciência, Tecnologia e Inovação em Saúde no Brasil, e permitirá que prioridades de pesquisa em saúde estejam em consonância com os princípios do Sistema Único de Saúde.

CONCLUSÕES: Há uma necessidade de mais estudos de avaliação para comparar o custo-benefício dos modelos de cuidados alternativos, de modo a melhorar a cobertura, pois a pesquisa pode tornar-se um instrumento para a mudança.

PALAVRAS-CHAVE: Enfermagem; Saúde mental; Pesquisa

\section{RESUMEN}

"Prioridades de investigación en salud mental y la transformación del modelo de atención"

CONTEXTO: En las dos últimas décadas, Brasil ha experimentado un proceso de transformación de su modelo de atención de salud mental, que se caracteriza por una reorientación de la atención centrada antes de la hospitalización por una lógica de la atención centrada en los servicios basados en la comunidad.

OBJETIVO(S): Este estudio tuvo como objetivo analizar la producción del conocimiento en Salud Mental por enfermeras de Brasil en relación con la prioridad temática de la Agenda de Salud Mental. METODOLOGÍA: Los datos se buscaran en artículos en idioma portugués en las bases de datos científicas indexadas. Para contextualizar el tema, una encuesta también se realizó sobre la distribución de los servicios de salud mental en Brasil.

RESULTADOS: De una búsqueda de la base de datos se encontraron 54 artículos. Entre ellos, cabe señalar que en Brasil existe una producción desigual con respecto al volumen de la investigación en enfermería de salud mental para cada región del país. Se propone que las enfermeras tratan de ajustar sus líneas de investigación priorizadas en la Agenda de Salud Mental. La articulación en torno a la Agenda es la acción más importante en la legitimación de este instrumento en la Política Nacional de Ciencia, Tecnología e Innovación en Salud en Brasil, y permitir que las prioridades de investigación en salud están en línea con los principios del Sistema de Salud.

CONCLUSIONES: Hay una necesidad de más estudios con el fin de comparar el costo-efectividad de los modelos alternativos de atención, para mejorar la cobertura, porque la investigación puede convertirse en un instrumento para el cambio.

\section{DESCRIPTORES: Enfermería; Salud mental; Investigación}

\section{ABSTRACT \\ "Priorities for research on mental health and the transforma- tion of care model"}

BACKGROUND: In the last two decades, Brazil has undergone a transformation of its model of mental health care, characterized by a redirection of care focused on hospitalization for a logic of attention focused on community-based services.

AIM: This study aimed to analyze the production of knowledge in Mental Health by Brazilian nurses in relation to the thematic priorities of the Mental Health Agenda.

METHODS: Data was sought in articles in Portuguese language in indexed scientific databases. To contextualize the issue, a survey was also made about the distribution of mental health services in Brazil.

RESULTS: From a search of the database 54 articles were found. Among them, it might be noted that in Brazil there is an uneven production with respect to the volume of research in nursing Mental Health for each region of the country. It is proposed that nurses seek to tune their research topics prioritized in the Mental Health Agenda. The linkage on the Agenda is the most important action in the legitimation of this instrument in National Policy for Science, Technology and Innovation in Health in Brazil, and will allow health research priorities that will be in line with the principles of the Public Health System.

CONCLUSIONS: We need more evaluation research to compare the cost-benefit of the models of alternative care, seeking the quality of coverage, because research may become crucial for a change.

\section{KEYWORDS: Nursing; Mental health; Research}

Submetido em 29-09-2014

Aceite em 22-12-2014

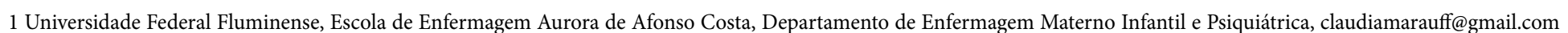

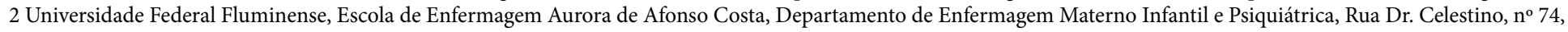
5ªndar, 24020-091 Niterói, Brasil, marcelapimentamuniz@gmail.com

3 Universidade Federal Fluminense, Escola de Enfermagem Aurora de Afonso Costa, 24020-091 Niterói, Brasil, tns.thiago@hotmail.com

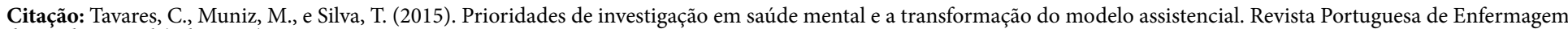
de Saúde Mental (Ed. Esp. 2), 107-112. 


\section{INTRODUÇÃO}

Nas duas últimas décadas o Brasil passou por um processo de transformação do seu modelo de atenção em saúde mental, caracterizado pelo redirecionamento de um cuidado antes centrado na internação hospitalar para uma lógica de atenção focada em serviços de base comunitária. Esse processo de mudança de modelo implica um conjunto de desafios para investigação científica, reforçando a importância da inclusão do tema saúde mental na Agenda de Prioridades de Pesquisa para o Sistema Único de Saúde (SUS). A Política Nacional de Saúde Mental no Brasil é essencialmente baseada na Declaração de Caracas (OMS, 1991), e prevê, sobretudo: o processo de desinstitucionalização do portador de transtorno psíquico, a reorientação da assistência na saúde mental para um modelo descentralizado e de base comunitária, a partir da redução gradual dos leitos psiquiátricos e com a progressiva ampliação da rede extra-hospitalar.

Neste sentido, os novos dispositivos de saúde mental que vêm sendo utilizados no Brasil são: os Centros de Atenção Psicossocial (CAPS); ações da saúde mental na Atenção Básica; os Serviços Residenciais Terapêuticos (SRT); os Centros de Convivência e Cultura; alguns Leitos de atenção integral em Hospitais Gerais e nos CAPS III (que funcionam 24 horas); o auxílio financeiro do Programa de Volta para Casa; e os Consultórios de Rua. Dentre os dispositivos disponíveis, o principal deles tem sido o CAPS, o qual constitui a principal estratégia do processo de Reforma Psiquiátrica no Brasil. De acordo com a Portaria no 336/GM de 2002, os CAPS são instituições destinadas a acolher os pacientes com transtornos mentais, estimular sua integração social e familiar, apoiá-los em suas iniciativas de busca da autonomia, oferecer-lhes atendimento médico e psicológico. Sua característica principal é a de buscar integrar o portador de sofrimento psíquico a um ambiente social e cultural concreto, designado como seu "território", o espaço da cidade onde se desenvolve a vida cotidiana desses usuários e de seus familiares (Brasil, 2002).

Os principais objetivos destes serviços tem sido: oferecer cuidados intermediários (entre o regime ambulatorial e a internação hospitalar) com ênfase na abordagem compreensiva e com suporte educacional, social, recreacional, reabilitação psicossocial e reinserção profissional; estimular a melhoria da qualidade de vida dos usuários e seus familiares através do autocuidado; e intervir junto à comunidade com ações educativas em saúde como forma de reduzir os danos sociais, psíquicos e físicos conseqüentes do uso de drogas.

\section{METODOLOGIA}

Buscou-se informações contidas em artigos, em língua portuguesa indexados em, bases de dados e Bibliotecas Virtuais em Saúde (BVS) como BIREME, SCIELO, MEDLINE, LILACS e BDENF a partir do descritor "Enfermagem Psiquiátrica". Usou-se como critérios de seleção os artigos estarem disponíveis na íntegra, escritos por brasileiros e cujos resumos evidenciassem concretamente que o foco principal de abordagem do artigo fosse relacionado à enfermagem psiquiátrica. $\mathrm{O}$ período considerado para a busca foi de janeiro de 2009 a julho de 2014.

Realizou-se uma análise dos 54 artigos científicos encontrados, levando-se em consideração as prioridades propostas pela agenda para pesquisas em saúde mental no Brasil. Dos artigos encontrados destacam-se os estudos desenvolvidos por Camatta (2009), Filizola, Teixeira, Milioni e Brechesi (2011), Mari, Bressan, Almeida-Filho, Gerolin, Sharan e Saxena (2006), Miranda, Clementino, Santos, Silva e Costa (2010), Moreira e Loyola (2010), Muniz, Tavares, Souza, Pacheco e Figueiredo (2014), Oliveira, Silva e Silva (2009), e Oliveira, Kestenberg e Silva (2013). Para contextualizar o tema, realizou-se um levantamento da distribuição dos serviços de saúde mental no Brasil, comparando-se regiões do país, serviços hospitalares e extra-hospitalares e desenvolvimento de pesquisas em enfermagem.

\section{RESULTADOS E DISCUSSÃO}

$\mathrm{O}$ atual cenário da saúde mental no Brasil tem apresentado como tendências: a reversão do modelo hospitalar para uma ampliação significativa da rede de atenção extra-hospitalar, de base comunitária; o entendimento das questões de álcool e outras drogas como problema de saúde pública e como prioridade no atual governo; e a ratificação das diretrizes do Sistema Único de Saúde (SUS) nas ações de saúde mental através da Lei Federal 10.216/01 e da III Conferência Nacional de Saúde Mental. Atualmente, $12 \%$ da população necessitam de algum atendimento em saúde mental, seja ele contínuo ou eventual, e 2,3\% do orçamento anual do SUS são destinados para a Saúde Mental (Ministério da Saúde, 2012). Neste sentido, alguns desafios precisam ser enfrentados, sobretudo no sentido de fortalecer às políticas de saúde voltadas para grupos de pessoas com transtornos mentais de alta prevalência e baixa cobertura assistencial, bem como implementar uma política de saúde mental mais eficaz no atendimento às pessoas que sofrem com a crise social, a violência e desemprego - determinantes sociais da saúde impactantes atualmente no Brasil. 
Para isto, o país tem apontado para a necessidade de consolidação e ampliação da rede de atenção de base comunitária e territorial (Gráfico 1) visando à promoção da reintegração social e cidadania. Portanto, o aumento dos recursos do orçamento anual do SUS para a Saúde Mental também deve ser encarado como importante desafio, a fim de se tornar factível o alcance das metas estabelecidas.

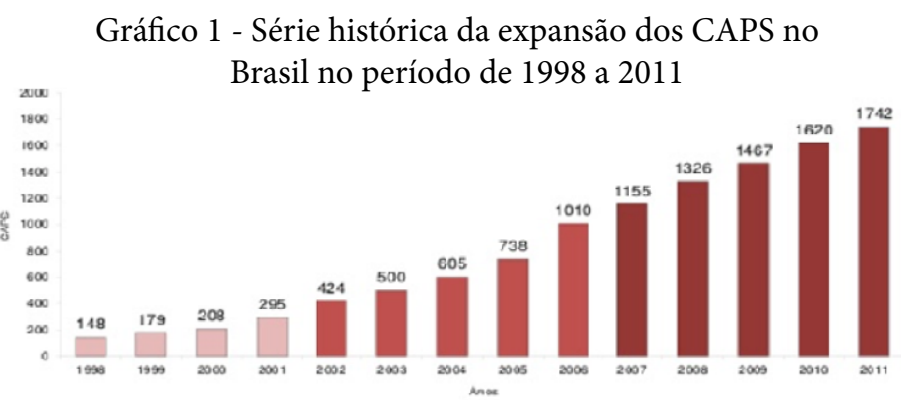

Fonte: Coordenação de saúde mental, álcool e outras drogas/DA$\mathrm{PES} / \mathrm{MS}$

As regiões do Brasil não demonstraram um equilíbrio na distribuição deste crescimento no número de CAPS. É mais expressivo o indicador da cobertura assistencial nas regiões Sul e Nordeste, que vêm apresentando os maiores indicadores de cobertura. A expansão do número de CAPS nas regiões Centro-oeste e Norte permanece como desafio a ser enfrentado nos próximos anos. Com relação à questão específica da expansão destes serviços na região Norte, o Ministério da Saúde (2012) aponta que, para melhor compreensão do problema é necessário que se ajuste o indicador CAPS/100.000 habitantes, devido às peculiaridades demográficas desta região. Já a região Sudeste, tem apresentado um ritmo de crescimento semelhante ao das regiões Centro-oeste e Norte, apesar de ter apresentado um patamar mais alto de cobertura no início do recorte histórico.

Este recorte pode ser melhor compreendido a partir da interpretação do gráfico 2, que mostra em seu comportamento a expansão dos CAPS em cada região do país.

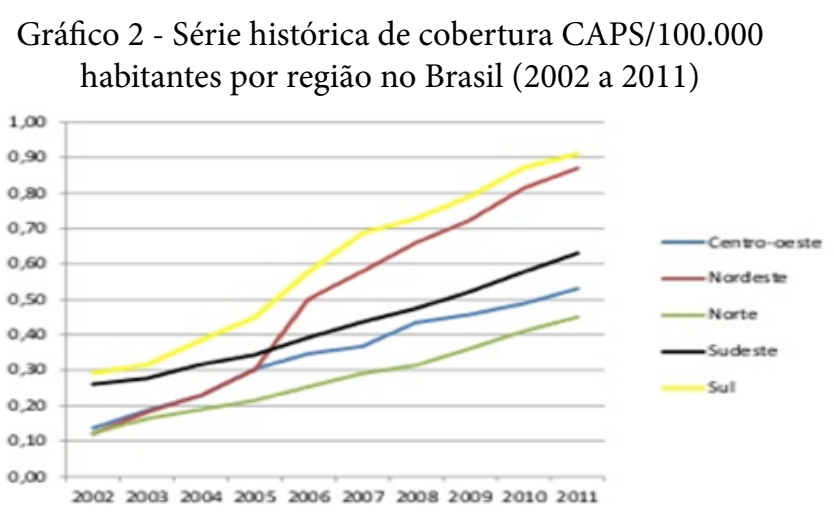

Fonte: Coordenação de saúde mental, álcool e outras drogas/DA$\mathrm{PES} / \mathrm{MS}$
Os SRT também apresentaram um movimento de expansão considerável no país, havendo um crescimento acentuado em 2011 devido ao processo de fechamento de grandes hospitais neste período (gráfico 3). O mesmo ocorreu com o Programa de Volta para Casa. O Ministério da Saúde aponta que em 2012 o Programa havia alcançado mais de 4 mil beneficiários em folha de pagamento (gráfico 4). Porém, tanto os SRT quanto o Programa de Volta para Casa precisam ampliar significativamente sua curva de crescimento, pois ainda há muitos usuários que não têm acesso a essas estratégias para a Reabilitação Psicossocial.

Gráfico 3 - Serviços Residenciais Terapêuticos por ano

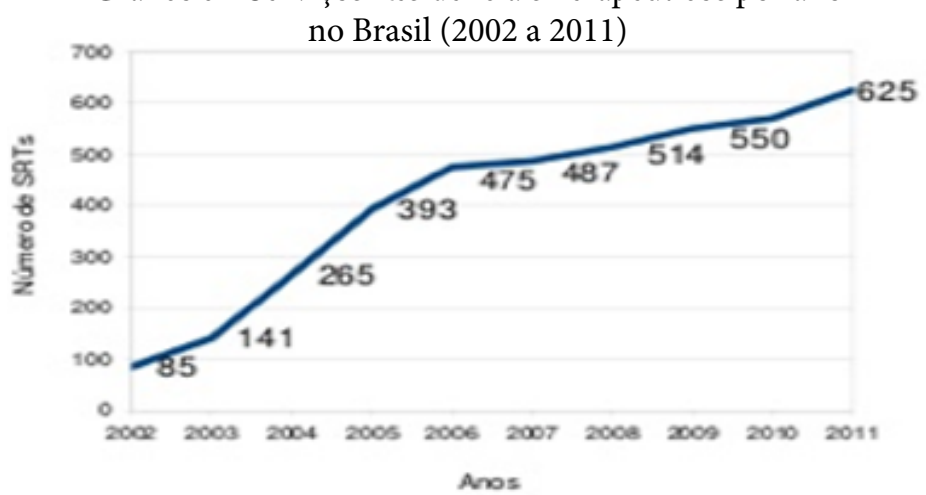

Fonte: Coordenação de saúde mental, álcool e outras drogas/DA$\mathrm{PES} / \mathrm{MS}$

Gráfico 4 - Beneficiários do Programa de Volta para casa por ano no Brasil (2003 a 2011)

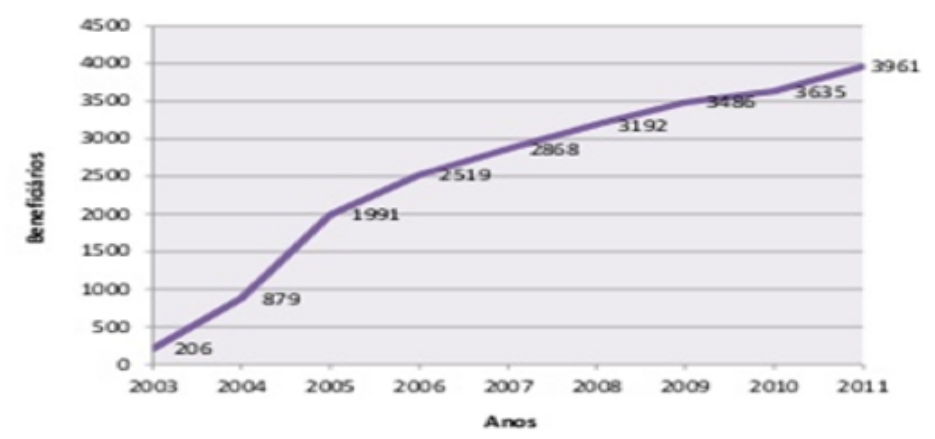

Fonte: Coordenação de saúde mental, álcool e outras drogas/DA$\mathrm{PES} / \mathrm{MS}$

Neste sentido, pode-se destacar a inversão que houve dos investimentos na atenção Psicossocial por parte do Ministério da Saúde. Se em 2002 aplicava-se apenas 24\% dos gastos na saúde mental em serviços extra-hospitalares, em 2011 essa porcentagem atingiu 71\%, contra 29\% investidos em serviços hospitalares (Brasil, 2012).

No entanto, verifica-se a partir da interpretação dos gráficos, que as novas modalidades terapêuticas apresentaram um ritmo de crescimento importante de 2002 a 2006. E de lá pra cá passaram a atingir ritmo de crescimento mais lento. 
Este fato relaciona-se com o próprio processo de discussão em torno da Reforma Psiquiátrica no Brasil que precisa ser reaquecido, retomando o debate a respeito da importância da ampliação de novas modalidades terapêuticas. Nesse contexto a presença da universidade é também requerida. É consenso que existe a necessidade de produção de conhecimentos que gerem mais saúde mental para a população, que auxiliem nas ações de expansão da rede de serviços, na melhora na qualidade da atenção e na redução da lacuna assistencial com ênfase na atenção primária, levando em conta os determinantes sociais e o enfrentamento do estigma na sociedade.

Neste sentido, propõe-se que os enfermeiros busquem sintonizar suas pesquisas às temáticas priorizadas na agenda de Saúde Mental. A articulação em torno da Agenda é a ação mais importante na legitimação deste instrumento na Política Nacional de Ciência, Tecnologia e Inovação em Saúde no Brasil, e permitirá que prioridades de pesquisa em saúde estejam em consonância com os princípios do SUS. Para isto, tem-se como pressuposto que as pesquisas visem respeitar as necessidades nacionais e regionais de saúde e estimular a produção de conhecimentos e bens materiais e processuais, sobretudo, nas áreas prioritárias, para o desenvolvimento das políticas sociais.

Destacam-se como tópicos a serem contemplados nas pesquisas que apresentam um potencial para suprir as lacunas de conhecimento identificadas na área de saúde mental: intervenções que possam reduzir a carga global da doença mental; estratégias mais eficientes de ampliar a oferta de serviços em saúde mental; atenção primária; bioética e direitos humanos das pessoas com transtornos mentais.

A produção de pesquisa em enfermagem de saúde mental no Brasil é marcada por uma produção desigual nas diferentes regiões do país, acompanhando o grau de desenvolvimento ecônomico e social dessas regiões. $\mathrm{O}$ gráfico 5 apresenta o total de artigos produzidos pelos pesquisadores em enfermagem do Brasil, exemplificando as disparidades regionais já mencionadas.

Gráfico 5 - Total de artigos publicados na área de enfermagem de saúde mental por região do Brasil (2010-2014)

\section{Total de artigos 54}

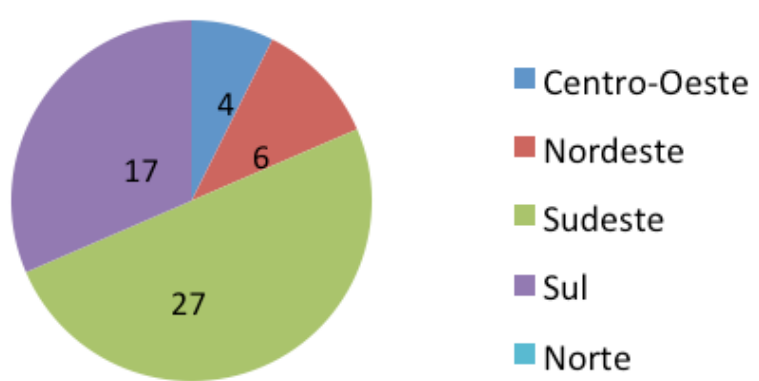

O foco das pesquisas realizadas por enfermeiros, segundo análise do material encontrado nas bases de dados investigadas, relaciona-se de um modo geral à agenda de pesquisa prioritárias em saúde mental no Brasil, predominando as pesquisas sobre as demandas de cuidado de enfermagem em saúde mental. A abordagem metodológica predominante é a qualitativa, desenvolvida em $74,1 \%$ dos estudos, seguida da quantitativa $24,1 \%$ e da quanti-qualitativa 1,8\% (Quadro 1).

Quadro 1 - Relação entre itens da agenda e foco da pesquisa realizada por enfermeiros no período de 2009 a 2014

\begin{tabular}{|c|c|c|}
\hline Itens da agenda & Foco da pesquisa em enfermagem & $\%$ \\
\hline $\begin{array}{l}\text { Enfoque } \\
\text { teórico- } \\
\text { metodológico }\end{array}$ & $\begin{array}{l}\text { Família e inclusão, internação e } \\
\text { cuidados de enfermagem; repre- } \\
\text { sentações sociais, profissionais e de } \\
\text { familiares sobre a doença mental; } \\
\text { cuidar em enfermagem psiquiátrica } \\
\text { hospitalar; vivência dos usuários } \\
\text { com a doença mental. }\end{array}$ & 20 \\
\hline $\begin{array}{l}\text { Magnitude, } \\
\text { dinâmica } \\
\text { e compreensão dos } \\
\text { agravos em saúde } \\
\text { mental }\end{array}$ & $\begin{array}{l}\text { Assistência psiquiátrica; internação } \\
\text { psiquiátrica involuntária; deman- } \\
\text { das de atendimento; depressão } \\
\text { em idosos; ensino do cuidado de } \\
\text { saúde mental; sofrimento psíquico } \\
\text { da equipe de enfermagem; con- } \\
\text { hecimento de enfermeiros sobre a } \\
\text { reforma psiquiátrica; diagnóstico de } \\
\text { enfermagem. }\end{array}$ & 27 \\
\hline $\begin{array}{l}\text { Organização e } \\
\text { avaliação de políti- } \\
\text { cas, programas e } \\
\text { serviços }\end{array}$ & $\begin{array}{l}\text { Reforma psiquiátrica, implantação, } \\
\text { percepção de alunos e profissionais; } \\
\text { cuidado de enfermagem ao paci- } \\
\text { ente com co-morbidade clínico- } \\
\text { psiquiátrica, família, autocuidado; } \\
\text { internação, acolhimento, alta; } \\
\text { consumo de psicofármacos. }\end{array}$ & 26 \\
\hline $\begin{array}{l}\text { Avaliação, } \\
\text { desenvolvimento e } \\
\text { aplicação de tecno- } \\
\text { logias }\end{array}$ & $\begin{array}{l}\text { Interconsulta psiquiátrica; serviços } \\
\text { psiquiátricos; cuidado em saúde } \\
\text { mental na estratégia saúde da famí- } \\
\text { lia; desinstitucionalização; papel do } \\
\text { enfermeiro, interação, intervenção } \\
\text { em crise; abuso de álcool e drogas; } \\
\text { projeto terapêutico. }\end{array}$ & 27 \\
\hline
\end{tabular}

O Quadro 2 apresenta os itens de prioridade da agenda para pesquisa em saúde mental pesquisados em menor número pelos enfermeiros.

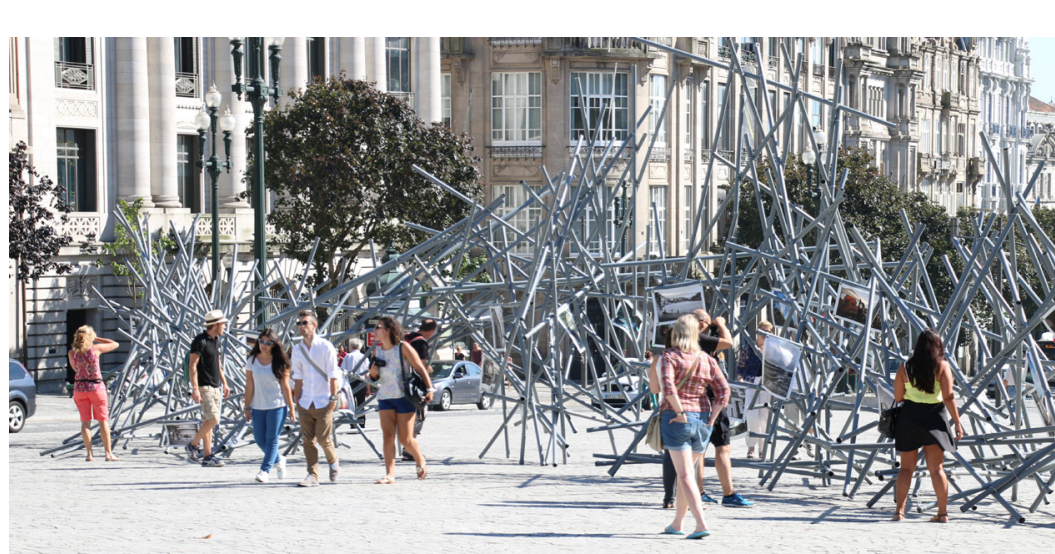


Quadro 2 - Temas da agenda que não têm sido pesquisados pelos enfermeiros de saúde mental no período de 2009 a 2014

\begin{tabular}{|l|l|}
\hline Itens da agenda & $\begin{array}{l}\text { A pesquisa em enfermagem } \\
\text { não tem contemplado }\end{array}$ \\
\hline $\begin{array}{l}\text { Enfoque } \\
\text { teórico-metodológico }\end{array}$ & $\begin{array}{l}\text { Gênero e etnia; violência e } \\
\text { implicações psicossociais; } \\
\text { ecologia social, urbana e rural; } \\
\text { saúde mental e religião. }\end{array}$ \\
\hline $\begin{array}{l}\text { Magnitude, dinâmica } \\
\text { e compreensão dos } \\
\text { agravos em saúde mental }\end{array}$ & $\begin{array}{l}\text { Indicadores de saúde mental; } \\
\text { carga global da doença mental; } \\
\text { fatores de risco e de proteção; } \\
\text { perfil epidemiológico; le- } \\
\text { vantamento exploratório de } \\
\text { aspectos demográficos e socio- } \\
\text { econômicos; fatores predispo- } \\
\text { nentes; transtornos mentais } \\
\text { pós-parto; saúde mental na } \\
\text { infância. }\end{array}$ \\
\hline $\begin{array}{l}\text { Organização e } \\
\text { avaliação de políticas, pro- } \\
\text { gramas e serviços }\end{array}$ & $\begin{array}{l}\text { Saúde mental, assédio moral e/ } \\
\text { ou sexual, trabalho e educação. }\end{array}$ \\
\hline $\begin{array}{l}\text { Avaliação, } \\
\text { desenvolvimento e } \\
\text { aplicação de tecnologias }\end{array}$ & $\begin{array}{l}\text { Novos métodos e técnicas de } \\
\text { investigação; pesquisas sobre } \\
\text { medicamentos para transtor- } \\
\text { nos mentais. }\end{array}$ \\
\hline
\end{tabular}

\section{CONCLUSÃO}

A mudança do modelo assistencial em saúde mental no Brasil desencadeou um conjunto de desafios para investigação científica, culminando com a inclusão do tema saúde mental na Agenda de Prioridades de Pesquisa para o SUS. Até o momento, a despeito dos enfermeiros desenvolverem pesquisas coadunadas com a agenda nacional de prioridades, persiste a necessidade de novos estudos serem realizados de forma mais equanime em todas as regiões do país. Ressalta-se a necessidade de mais estudos de avaliação, para comparar o custo-benefício dos modelos de cuidados alternativos - uma forma de melhorar a cobertura pelo uso da própria pesquisa como um instrumento para a mudança.

Os estudos analisados indicam importantes avanços nos cuidados desenvolvidos com a pessoa portadora de sofrimento mental, o significado que assume a doença mental junto à família e a relação com os novos dispositivos assistenciais em saúde. Contudo, é necessário que a sociedade, o governo e a universidade ampliem o debate em torno de novas estratégias de atenção em saúde mental e também possa investigar sua eficácia.

Tangente a isto, concluiu-se a relevância dos enfermeiros contemplarem a temática dos desafios enfrentados na clínica e na política de saúde mental.
Por isto, recomenda-se que os serviços de saúde e as universidades intensifiquem parcerias para que conjuguem formas de cuidar que atendam ao imperativo ético da Reforma Psiquiátrica, e que se qualifique a infra-estrutura para apoio à pesquisa no país.

\section{REFERÊNCIAS BIBLIOGRÁFICAS}

Camatta, M (2009). The life experience of families concerning the work of the mental health professionals: A phenomenological study. Online Brazilian Journal of Nursing, 8(2), 16-28. doi: 10.5935/1676-4285.20092183

Filizola, C. L. A, Teixeira, I. M. C., Milioni, D., e Brechesi, P. S. C. (2011). Saúde mental e economia solidária: A família na inclusão pelo trabalho. Revista da Escola de Enfermagem da USP, 45(2), 418-425. doi: 10.1590/ S0080-62342011000200017

Mari, J. J., Bressan, R. A., Almeida-Filho, N., Gerolin, J., Sharan, P., e Saxena, S. (2006). Pesquisa em saúde mental no Brasil: Políticas, infra-estrutura, financiamento e recursos humanos. Revista de Saúde Pública, 40(1), 161-169.

Ministério da Saúde (2002). Portaria no 336. Brasília: Ministério da Saúde.

Ministério da Saúde. Secretaria de Atenção à Saúde. Departamento de Ações Programáticas Estratégicas Coordenação Geral de Saúde Mental, Álcool e Outras Drogas (2012). Saúde Mental em Dados. Brasília: Ministério da Saúde.

Ministério da Saúde (2008). Informes técnicos institucionais. Saúde define prioridades de pesquisa. Revista de Saúde Pública, 42(5), 974-977.

Miranda, F. A. N., Clementino, F. S., Santos, R. C. A., Silva, M. B., e Costa, T. S. (2010). Percepção de alunos de graduação em enfermagem sobre o processo da reforma psiquiátrica no Brasil. Revista Enfermagem UERJ, 18(2), 235-240.

Moreira, L. H. O., e Loyola, C. M. D. (2010). Internação psiquiátrica involuntária: Implicações para a relação enfermagem/paciente. Revista Enfermagem UERJ, 18(4), 632-637. 
Muniz, M. P., Tavares, C. M. M., Souza, Â. C., Pacheco, C. K. R., e Figueiredo, L. (2014). Desvendando o projeto terapêutico de enfermagem em saúde mental: Um relato de experiência. Revista de Pesquisa: Cuidado é Fundamental On line, 6(1), 132-140. doi: $10.9789 / 2175-5361.2014 v 6 n 1 p 132$

Oliveira, E. B., Kestenberg, C. C. F., e Silva, A. V. (2013). Modelo intervenção em crise: A saúde mental como abordagem do cuidado de enfermagem no hospital geral. Revista de Pesquisa: Cuidado é Fundamental On line, 5(1), 3176-3184. doi: 10.9789/2175-5361.2013v5n1p3176
Oliveira, F. B., Silva, K. M. D., e Silva, J. C. C. (2009). Percepção sobre a prática de enfermagem em Centros de Atenção Psicossocial. Revista Gaúcha de Enfermagem, 30(4), 692-699.

Organização Mundial de Saúde (1991). Declaração de Caracas. Princípios de para proteção de pessoas acometidas de transtorno mental, e melhoria de assistência à saúde. Caracas: Organização Mundial de Saúde.

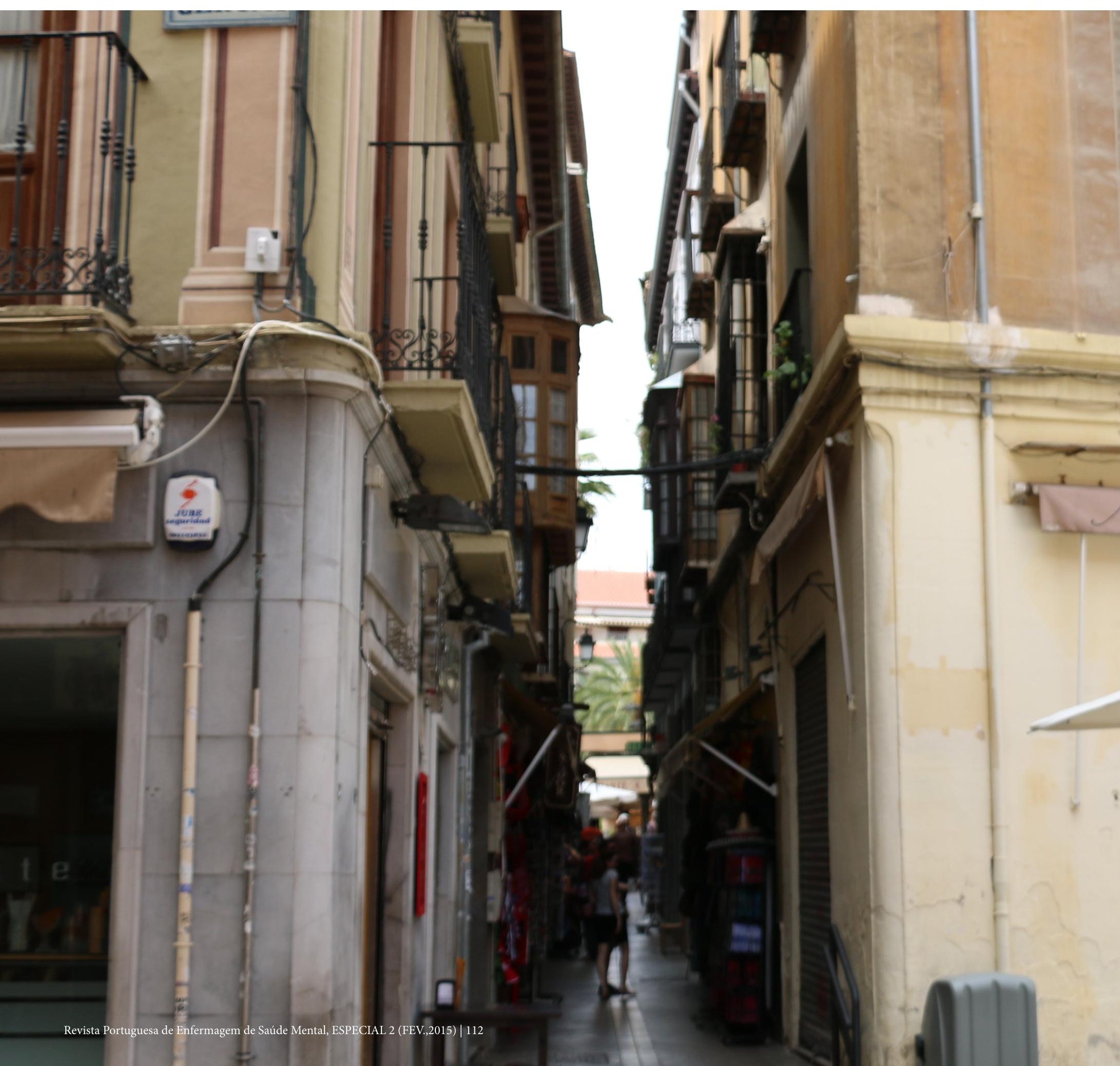

